\title{
A practical guide to the interpretation of PK/PD profiles of longer-acting analogue insulins. Part two: Insulin degludec vs. insulin glargine U300
}

\author{
Oppel BW Greeffa, Jacob John van Tonder ${ }^{b}$, Kershlin Naiduc, Alicia McMaster ${ }^{d *}$, Alet van Tonderd and Rashem Mothilald \\ ${ }^{a}$ Department of Pharmacology, University of Pretoria, Pretoria, South Africa \\ ${ }^{b}$ Triclinium Clinical Development (Pty) Ltd, Centurion, South Africa \\ 'Department of Internal Medicine, Chris Hani Baragwanath Academic Hospital, University of the Witwatersrand, Johannesburg, South Africa \\ ${ }^{d}$ Sanofi-Aventis South Africa (Pty) Ltd, Midrand, South Africa \\ *Corresponding author, email: alicia.mcmaster@sanofi.com
}

\begin{abstract}
Glucose clamp studies form an integral part of the early development of insulin therapies. Data generated in these studies are used to establish pharmacokinetic (PK) and pharmacodynamic (PD) profiles of the agents, but methodological differences confound comparison of results from different glucose clamp studies. The first part of this series on glucose clamp studies discussed practical tips for the interpretation of glucose clamp studies. The second part of the series compares the PK/PD profiles of longer-acting basal analogue insulins, insulin degludec (IDeg) and insulin glargine U300 (Gla-300). The patient populations for glucose clamp studies with these analogue insulins differ, and therefore direct comparison of the data is not always possible. The maximum duration of action of IDeg is reported as $42 \mathrm{~h}$ and that of Gla-300 as $36 \mathrm{~h}$, translating to $24 \mathrm{~h}$ coverage. The plasma insulin concentration of IDeg is 56 times that of Gla-300. Results from phase III clinical trials for these analogue insulins confirm the predictability and low withinsubject variability observed in glucose clamp studies. Insight into the PK/PD profiles of longer- acting basal analogue insulins allows the treating physician to utilise these characteristics to optimise the treatment of their patients with diabetes.
\end{abstract}

Keywords: analogue insulins, glucose clamp, time-action profile, pharmacokinetics, pharmacodynamics

Republished with permission: Journal of Endocrinology, Metabolism and Diabetes of South Africa 2018; 23(2):46-51.

https://doi.org/10.1080/16089677.2017.1407097

\section{Introduction}

Exogenous insulin is used to treat hyperglycaemia in selected patients diagnosed with diabetes mellitus (DM). In order to mimic physiological insulin secretion, treatment with long- acting basal insulins, as well as rapid-acting insulins, may be required.'

The ideal longer-acting analogue insulin will display flat, peakless pharmacokinetic (PK) and pharmacodynamic (PD) profiles with limited inter- and intra-individual variability. The time-activity profile of an analogue insulin is determined with a glucose clamp study. A glucose clamp study measures the amount of glucose required to maintain the plasma glucose concentration at a predetermined level referred to as the clamp target, in terms of the glucose infusion rate (GIR), which provides a way to describe the PD profile of the study insulin. The pharmacokinetic profile of the study insulin is determined by the plasma insulin concentration over time, as observed during the glucose clamp study.

A major concern with the use of exogenous insulin is the potential for causing hypoglycaemia. The PK/PD properties of an analogue insulin, as determined during early clinical development, can be used to predict the potential, be it high or low, of the insulin to induce hypoglycaemia. These results are later confirmed in pivotal clinical trials. Ideally, an analogue insulin should present with a peakless PK profile, which translates into constant and predictable plasma insulin concentration and reduced risk for hypoglycaemia. ${ }^{2}$ In contrast, high variability in the PK profile indicates a higher probability of hypoglycaemia episodes and difficulty in titrating a patient's exogenous insulin treatment to a required glucose target.

Since insulin production became possible, numerous insulin preparations have been developed that necessitated multiple daily administrations due to insufficient duration of action to provide basal insulin coverage for 24 h. ${ }^{3}$ The first long-acting basal analogue insulin produced was insulin glargine, referred to as Gla-100. During the development of Gla-100 an amino acid substitution was made at position A21, glycine for asparagine. ${ }^{4}$ This amino acid substitution improved the stability of the molecule and extended its duration of action. The duration of action of Gla-100 is reported to be $24 \mathrm{~h}^{3}$, translating into oncedaily administration. Gla-100 also shows a smaller gradient in its PK profile than NPH or regular human insulin, as well as reduced variability.

However, to accurately replicate physiological basal insulin coverage, duration of action exceeding $24 \mathrm{~h}$ is required with a peakless PD (time-activity) profile to further minimise the risk of hypoglycaemia. ${ }^{5}$ To address the current unmet need in basal analogue insulins, two longer-acting insulin analogues have been developed: insulin degludec (IDeg) and insulin glargine U300 (Gla-300). As part of the clinical development plan of 
these analogue insulins, glucose clamp studies were performed to determine their PK and PD profiles. While no head-to-head glucose clamp data are currently available comparing the PK/ PD profiles of IDeg and Gla-300, the results of numerous glucose clamp studies have been published for these two molecules separately.

Following on from the practical tips for interpreting the results of glucose clamp studies in part one of this series, the PK/PD profiles of IDeg and Gla-300 will now be discussed.

\section{Published glucose clamp data on IDeg and Gla- 300}

In the previous part of this series on the interpretation of glucose clamp studies, six main aspects of glucose clamp studies to consider when interpreting results were suggested: (1) clamp methodology, (2) duration of glucose clamp study, (3) study population, (4) dose of study insulin, (5) glucose infusion rate and (6) plasma insulin concentration. Following is a practical application of these points to compare results published from glucose clamp studies of IDeg and Gla-300.

\section{Clamp methodology}

Glucose clamp studies can be performed manually, where plasma glucose levels are measured in intervals of several minutes and the glucose infusion rate is manually adjusted to maintain clamp target, or with the use of a Biostator MTB (Medizintechnik, Amstetten, Germany), allowing for determination of glucose levels every minute and automated adjustment of the glucose infusion rate. The use of the Biostator in glucose clamp studies eliminates potential biased adjustment of the glucose infusion rate and allows for standardisation of study conditions. ${ }^{6}$ Published glucose clamp studies of IDeg ${ }^{7-9}$ and Gla-300 $0^{5,10-12}$ made use of a Biostator. As is standard practice in glucose clamp studies using Biostators, study participants in all glucose clamp studies with IDeg and Gla-300 were connected to the Biostator several hours prior to initiation of the clamp procedure as part of a stabilisation period.

\section{Duration of glucose clamp study}

The clinically appropriate end of the glucose clamp study is defined as the point in time when clamp target glucose level is maintained at GIR $=0 \mathrm{mg} / \mathrm{kg} / \mathrm{min} .{ }^{13}$ Therefore, the action of the study insulin has diminished to such an extent that blood glucose levels remain at clamp target without infusion of glucose. However, the duration of action of the study insulin may exceed the duration of the glucose clamp study. The duration of glucose clamp studies for longer-acting basal analogue insulins often exceeds $24 \mathrm{~h}$. Indeed, the duration of glucose clamp studies performed for IDeg ranged from $24 h^{7,8}$ to $42 h^{9}$ and that of Gla-300 from $24 h^{12}$ to 36 h., ${ }^{5,10,11}$

Based on the results of the glucose clamp study with the longest duration, the half-life of IDeg has been reported as exceeding $24 \mathrm{~h}$ and its duration of action as more than $42 \mathrm{~h} .{ }^{9}$ The GIR had not reached $0 \mathrm{mg} / \mathrm{kg} / \mathrm{min}$ at the $42 \mathrm{~h}$ mark and thus it was concluded that the duration of action of IDeg exceeded the duration of the clamp study. ${ }^{9}$ During this study, the mean half- life of the three concentrations of IDeg used was calculated as $25.4 \mathrm{~h} .{ }^{9}$

The duration of glucose clamp studies for Gla-300 has not extended beyond $36 \mathrm{~h}$. Of the three studies with a duration of $36 \mathrm{~h}$, only one conclusively showed GIR $=0 \mathrm{mg} / \mathrm{kg} / \mathrm{min}$ at the $36 \mathrm{~h}$ mark. ${ }^{10}$ It can be argued that as GIR $=0 \mathrm{mg} / \mathrm{kg} / \mathrm{min}$ had not been reached in the other glucose clamp studies with $36 \mathrm{~h}$ duration ${ }^{11,12}$, the duration of action of Gla-300 may exceed the duration of the clamp studies.

The longer-acting basal analogue insulins, IDeg and Gla-300, will provide basal insulin coverage exceeding $24 \mathrm{~h}$. The extended duration of action may translate into greater flexibility in dosing than was possible with previous basal analogue insulins. However, as the true duration of action of these insulins cannot be accurately determined, it is not possible to estimate the total plasma insulin concentration from these results.

\section{Study population}

The patient population for glucose clamp studies can be selected to limit the effect of endogenous insulin secretion on PK/PD parameter estimations. Glucose clamp studies performed in a Type $1 \mathrm{DM}$ patient population is considered to be ideal as the effect of endogenous insulin secretion is eliminated. However, as the real-world patient population mainly consists of patients diagnosed with Type $2 \mathrm{DM}$, it is valuable to obtain PK/PD data in a Type 2 DM patient population as well. ${ }^{13,14}$ In fact, true PK/PD estimates for the larger patient population can therefore only be determined from studies based on a truly representative study population.

In order to confirm low residual endogenous insulin secretion in the glucose clamp population of patients diagnosed with Type $1 \mathrm{DM}$, the fasting serum C-peptide concentration is often determined. C-peptide is released upon the conversion of preinsulin to insulin and therefore provides an accurate measure of endogenous insulin secretion. ${ }^{15}$ The fasting serum C-peptide concentration for participants in IDeg glucose clamp studies was below $0.3 \mathrm{nmol} / \mathrm{l} .{ }^{8,9}$ Similarly, glucose clamp studies for patients diagnosed with Type 1 DM for Gla-300 required a fasting serum C-peptide concentration below $0.3 \mathrm{nmol} / \mathrm{I}^{5,10-12}$ Therefore, the potential for endogenous insulin to interfere in these glucose clamp studies was negligible.

In order to control the effect of endogenous insulin in patients diagnosed with Type 2 DM, low fasting C-peptide levels are often required for inclusion of patients diagnosed with Type 2 diabetes in glucose clamp studies. ${ }^{16}$ As such, inclusion criteria for patients diagnosed with Type $2 \mathrm{DM}$ for IDeg included a fasting serum C-peptide concentration below $1.0 \mathrm{nmol} / \mathrm{l}$. Glucose clamp studies for Gla-300 have not been conducted in patients diagnosed with Type $2 \mathrm{DM}$. These levels of fasting serum C-peptide would eliminate the inclusion of patients with endogenous insulin secretion, which may confound results obtained.

One way to address the issue of inter-individual variability in trials is to use a crossover study design. This design is commonly used 
whereby a single patient population is used to investigate the PK/ PD parameters of the study and comparator insulin. This design reduces the number of volunteers required and yields more comparable results between the study and comparator insulin as the degree of insulin resistance, rate of insulin metabolism and other patient-specific factors remain constant between study groups. This type of design provides less confounded estimates of PK/PD parameters, which is why it was used in all of the glucose clamp studies discussed here, except one. ${ }^{8}$

\section{Dose of study insulin}

The dosages of study insulin and conditions for investigating the time-action profile of longer-acting basal analogue insulins should reflect those of the general treatment population. Therefore, the use of reasonable insulin doses at steady-state conditions are regarded as the most appropriate for use in glucose clamp studies. ${ }^{13}$

Multiple concentrations of study insulin are usually investigated during glucose clamp studies. PK/PD profiles of IDeg were determined at concentrations of $0.4,0.6$ and $0.8 \mathrm{IU} / \mathrm{kg}$ for patients diagnosed with Type $2 \mathrm{DM}^{7}$ or Type $1 \mathrm{DM}^{9}$ and $0.4 \mathrm{IU} / \mathrm{kg}$ for patients diagnosed with Type $1 \mathrm{DM} .{ }^{8} \mathrm{Gla}-300$ was used at concentrations of 0.4 and $0.6 \mathrm{IU} / \mathrm{kg}^{10-12}$ with the European population in the Shiramoto study also receiving Gla-300 at $0.9 \mathrm{lU} / \mathrm{kg}^{11}$

It was recently reported that the average daily basal insulin dose of patients diagnosed with T2DM is $0.41 \pm 0.43 \mathrm{IU} / \mathrm{kg}^{17}$ suggesting that the doses of IDeg and Gla-300 used in glucose clamp studies were clinically relevant. However, it must be considered that in the general treatment population titration and adjustment of insulin doses occur frequently and therefore the fixed concentrations of study insulin selected for the glucose clamp study may not accurately reflect clinical practice. ${ }^{9}$

As the conditions of the study population in glucose clamp studies should reflect the conditions in the general treatment population, the use of steady-state conditions is recommended as this most accurately replicates the use of long-acting analogue insulins in the treatment population. ${ }^{13}$ Steady-state conditions were used for glucose clamp studies investigating the PK/PD profile of IDeg. ${ }^{7-9}$ However, glucose clamp studies for Gla-300 were performed after a single subcutaneous dose ${ }^{11}$ and under steady-state conditions. ${ }^{5,10,12}$

Shiramoto et al. reported the results of glucose clamp studies performed after a single subcutaneous dose of Gla-300 in Japanese patients diagnosed with Type 1 DM. ${ }^{11}$ In this study a single subcutaneous dose of 0.4 or $0.6 \mathrm{IU} / \mathrm{kg}$ Gla-300 was administered after an overnight fast. A maximum insulin concentration $\left(C_{\max }\right)$ of $10.9 \mu \mathrm{U} / \mathrm{ml}$ was recorded over the $36 \mathrm{~h}$ clamp period after a single administration of $0.4 \mathrm{lU} / \mathrm{kg}$ of Gla300. ${ }^{11}$ However, where glucose clamp studies were performed in patients diagnosed with Type $1 \mathrm{DM}$ at steady-state conditions, i.e. after daily administration of $0.4 \mathrm{IU} / \mathrm{kg}$ Gla-300 for 8 days, $\mathrm{C}_{\max }$ was recorded as $18.1 \mu \mathrm{U} / \mathrm{ml}$ over the $36 \mathrm{~h}$ clamp period, ${ }^{10}$ almost double the concentration reported in the single-dose study. In the general treatment population it is more likely that patients will receive daily doses of insulin, and therefore PK/ PD parameters reported at steady-state conditions are more clinically relevant.

\section{Glucose infusion rate}

The GIR is adjusted according to measured blood glucose values to maintain blood glucose levels at predetermined clamp targets. GIR is the main PD endpoint determined during glucose clamp studies. Fluctuation in the GIR observed during the clamp study is indicative of intra-individual variation in response and may translate into unpredictable intra-individual response. Fluctuation in GIR is often reported as the coefficient of variation (CV). ${ }^{16}$

From a clinical perspective, intra-individual variation may be the most relevant parameter. Intra-individual variation affects the predictability of an individual's response to treatment. Unfortunately, not all studies report on these parameters.

Intra-individual variation of $20 \%$ in total GIR was reported for a clamp study performed with IDeg at a concentration of $0.4 \mathrm{IU} / \mathrm{kg}$ under steady-state conditions in a study population of 54 Type $1 \mathrm{DM}$ patients. ${ }^{8}$ After a $24 \mathrm{~h}$ euglycemic clamp study in 50 Type $1 \mathrm{DM}$ patients receiving $0.4 \mathrm{lU} / \mathrm{kg}$ of Gla-300, an intra-individual variation of $34.8 \%$ in total GIR was reported under steady-state conditions. ${ }^{12}$ Data thus indicate that a lower intra-individual variability, and therefore potentially a more reliable patient response, may be achieved with IDeg in a Type 1 DM treatment population. However, this still requires confirmation with results from pivotal clinical studies.

Inter-individual variability predicts the treatment population's response to treatment. Unfortunately, the inter-individual variability was not reported in clamp studies of IDeg performed in patients diagnosed with Type $1 \mathrm{DM}$. Inter-individual variability in total GIR was reported as $43.2 \%$ under steady-state conditions in a study population of Type $1 \mathrm{DM}$ patients treated with $0.4 \mathrm{IU} / \mathrm{kg}$ of Gla-300.12

Inter-individual variability for IDeg is available for a Type 2 DM treatment population: the total GIR of glucose clamp studies performed with IDeg at a concentration of $0.4 \mathrm{IU} / \mathrm{kg}$ has reported an inter-individual variability of $67.9 \%$ under steady-state conditions in a study population of patients diagnosed with Type 2 DM. ${ }^{7}$ However, data on the inter-individual variability in a Type 2 DM treatment population for Gla-300 are not currently available. As the inter-individual variability reported was not obtained in the same treatment population, accurate comparison of the data is not possible. As these results are not available, there is no indication of the variation expected between patients being titrated in a similar manner and therefore the reliability of a physician achieving the correct dose for a specific patient if he/ she uses the same approach for all patients.

A caveat in the design of glucose clamp studies is the use of rapid-acting insulin when establishing clamp target. To establish the euglycemic clamp target, most patients require a glucose infusion. However, when plasma glucose concentrations become hyperglycaemic, rapid-acting insulin analogues or human insulin 
may be used to re-establish the euglycemic clamp levels. For IDeg clamp studies rapid-acting human regular insulin was used up to 10 min before dosing of the study insulin, ${ }^{7-9}$ while Gla-300 studies made use of insulin glulisine. The duration of action of human regular insulin is estimated at seven hours ${ }^{18}$ and that of insulin glulisine at one to two and a half hours. ${ }^{3}$

As the infusion of rapid-acting human regular insulin and insulin glulisine were terminated shortly prior to initiation of the glucose clamp, these insulins may affect the glucose infusion rate required to maintain the clamp target. For studies using regular human insulin, this effect may influence the GIR for up to eight hours, while the GIR of studies using insulin glulisine may be affected for up to two and a half hours.

The total GIR over clamp period is often expressed as the GIR area under the curve (AUC) or GIR-AUC. Where the duration of the clamp is $24 \mathrm{~h}$, the GIR of the study can be visualised at sixhourly intervals, allowing for an overall view of the distribution of the study insulin effect over $24 \mathrm{~h}$. The GIR-AUC of IDeg and Gla- 300 over six-hourly intervals as proportions of the total GIRAUC is shown in Figure 1.

The PD profiles of both IDeg and Gla-300 demonstrate duration of action exceeding $24 \mathrm{~h}$ with minimal fluctuation in GIR between the different six-hourly intervals. A slight increase in GIR is observed for IDeg between $6 \mathrm{~h}$ and $12 \mathrm{~h}$ and for Gla-300 between $0 \mathrm{~h}$ and $6 \mathrm{~h}$. It must be considered, however, that the insulins used to establish the glucose clamp may still be affecting the GIR during the first hours of the glucose clamp therefore potentially confounding the observed GIR in the interval following the administration of short-acting insulin. Therefore, the use of insulin glulisine in establishing clamp target may contribute to the slight fluctuation observed in the GIR of Gla300 in the first six-hourly interval. For clamp studies with IDeg the effect of human regular insulin used to establish the clamp target may affect GIR for the first seven hours of the clamp study.

Recently the results of a euglycemic glucose clamp study comparing Gla-300 and IDeg were presented at the Diabetes Technology Society conference. The study was performed in

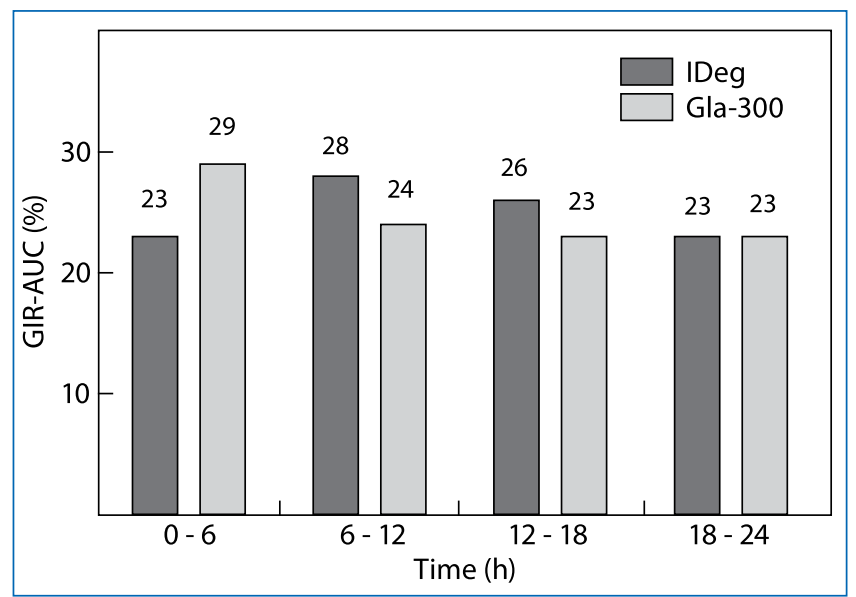

Notes: The glucose clamp studies were performed at a steady-state of IDeg and Gla-300. A dose of $0.4 \mathrm{IU} / \mathrm{kg}$ was used for both longer-acting basal analogue insulins.

Figure 1: Diurnal fluctuations in the glucodynamics of IDeg 9 and Gla-300. ${ }^{12}$
Table 1: Comparison of published glucose clamp results of insulin degludec (IDeg) and insulin glargine U300 (Gla-300)

\begin{tabular}{|c|c|c|}
\hline Factor & Insulin degludec & $\begin{array}{l}\text { Insulin glargine } \\
\qquad 300\end{array}$ \\
\hline Clamp methodology & Biostator 7,8 & Biostator ${ }^{12}$ \\
\hline Duration of clamp study & $\begin{array}{l}24 h^{8} \\
26 h^{7} \\
42 h^{9}\end{array}$ & $\begin{array}{c}24 h^{12} \\
36 h^{5,10,11}\end{array}$ \\
\hline Study population & $\begin{array}{l}\text { Type } 1 \mathrm{DM}^{8,9} \\
\text { Type } 2 \mathrm{DM}^{7}\end{array}$ & Type $1 \mathrm{DM}^{5,10-12}$ \\
\hline Dose of study insulin & $\begin{array}{c}0.4,0.6 \\
0.8 \mathrm{IU} / \mathrm{kg}^{7-9}\end{array}$ & $\begin{array}{c}0.4,0.6 \\
0.9 \mathrm{IU} / \mathrm{kg} / \text { day }^{5,10-12}\end{array}$ \\
\hline Steady state & Yes $^{7-9}$ & $\begin{array}{l}\text { Yes }^{5,10,12} \\
\mathrm{No}^{11}\end{array}$ \\
\hline $\begin{array}{l}\text { Maximum plasma insulin } \\
\text { concentration (study } \\
\text { insulin at } 0.4 \mathrm{lU} / \mathrm{kg} \text { ) }\end{array}$ & $\begin{array}{l}\text { Approx. } \\
833.3 \mu \mathrm{U} / \mathrm{ml}^{9}\end{array}$ & $\begin{array}{c}15 \mu \mathrm{U} / \mathrm{ml}^{10,12} \\
10.9 \text { and } 8.9 \mu \mathrm{U} / \mathrm{ml}^{11}\end{array}$ \\
\hline
\end{tabular}

a population of patients diagnosed with Type $1 \mathrm{DM}$, under steady-state conditions of Gla-300 or IDeg at $0.4 \mathrm{IU} / \mathrm{kg}$. Results indicated that Gla-300 resulted in less within-day variability in glucodynamics than IDeg. ${ }^{19}$ Complete results of the study have not yet been published.

\section{Plasma insulin concentration}

In order to determine the pharmacokinetic profile of longeracting basal analogue insulins, the plasma insulin concentration of the study insulin is measured during the glucose clamp study. The assays used to determine these concentrations are highly sensitive and specific to the study insulin, and therefore the concentration of residual endogenous insulin, in the case of a study population of patients diagnosed with Type $2 \mathrm{DM}$, or other insulins used to establish the clamp target should not interfere.

IDeg is highly plasma protein bound due to acylation of the $B$ chain. ${ }^{20,21}$ Its high binding affinity for serum albumin translates into an extended duration of action, ${ }^{20}$ but may confound accurate quantification of plasma insulin concentrations. Using currently available immunoassays only the concentration of the free, unbound fraction of IDeg can be quantified.

The method used for the determination of serum IDeg concentrations was not discussed. ${ }^{8}$ In one glucose clamp study the serum IDeg concentration was quantified using a specific sandwich enzyme-linked immunosorbent assay (ELISA) using a mouse monoclonal antibody specific for human insulin..$^{7,9}$ The study population of one of these studies consisted of patients diagnosed with Type 2 DM with confirmed fasting serum C-peptide concentrations below $1.0 \mathrm{nmol} / \mathrm{l}$, effectively excluding participants with residual endogenous insulin secretion. It must be considered that this method is suited to quantify only the free, and pharmacologically active, IDeg, which may result in underestimation of total IDeg plasma concentration. ${ }^{22}$

Plasma serum concentrations of Gla-300 and its metabolites were quantified using a radio-immunosorbent assay ${ }^{10-12}$ and liquid chromatography coupled to tandem mass spectrometry., ${ }^{5,10,11}$ Both methods had a lower limit of quantitation of $30 \mathrm{pmol} / \mathrm{I}^{10-12}$ or less. ${ }^{5}$ As Gla-300 is not known to be highly protein bound, 
quantitation using immunoassays is expected to accurately reflect the total plasma concentration. ${ }^{22}$

During a glucose clamp study with IDeg at $0.4 \mathrm{IU} / \mathrm{kg}$ under steady-state conditions a $C_{\max }$ of approximately $833.3 \mu \mathrm{U} / \mathrm{ml}$ was reported. ${ }^{9}$ Where Gla-300 was used at $0.4 \mathrm{IU} / \mathrm{kg}$ under steadystate conditions, $C_{\max }$ of approximately $15 \mu \mathrm{U} / \mathrm{ml}$ was reported..$^{10,12}$

This distinct difference between the maximum plasma concentrations of IDeg and Gla-300 can be attributed to the plasma protein bound nature of IDeg if the levels reflect total plasma insulin concentration. Fasting plasma concentration of endogenous insulin has been reported as $23-36 \mu \mathrm{U} / \mathrm{ml} .{ }^{23} \mathrm{An}$ approximate increase of 30-fold in plasma insulin concentration is thus observed after administration of IDeg in comparison with endogenous insulin.

The results from glucose clamp studies of IDeg and Gla-300 are summarised in Table 1.

From the data obtained from glucose clamp studies for the two longer-acting analogue insulins, insulin degludec and insulin glargine U300, it is clear that there are distinct differences between the insulins. Insulin degludec, due to the acetylation of the beta- chain, is highly plasma protein bound and therefore has an extended duration of action of up to $42 \mathrm{~h} .{ }^{9}$ This also results in high plasma levels of IDeg determined during glucose clamp studies. Gla-300 is similar in structure to insulin glargine U100, but has a distinct PK/PD profile with duration of action of up to 36 h. $^{10}$

Both of these analogue insulins provide flat PD profiles, more predictable responses and basal insulin coverage longer than $24 \mathrm{~h}$, which has not been possible with current insulin treatments. These characteristics have been associated with lower incidence of hypoglycaemia. ${ }^{16}$ However, effects on hypoglycaemia, difference in dosing frequency and intervals of these insulins can only be demonstrated conclusively through rigorous large clinical studies.

The efficacy and safety of IDeg and Gla-300 have been investigated in randomised, controlled phase 3 studies and confirm the clinical benefits of the longer-acting insulins in comparison to insulin glargine U100. The BEGIN trial programme demonstrated that IDeg, when compared with insulin glargine U100, is non-inferior with regard to glycaemic control as measured by $\mathrm{HbA} 1 \mathrm{C}$ and a reduction in the rate of nocturnal, confirmed hypoglycaemia was also observed in heterogeneous populations of patients diagnosed with diabetes. ${ }^{24-26}$ When Gla300 was compared with insulin glargine U100 in the EDITION trial programme, non-inferiority with regard to glycaemic control as measured by $\mathrm{HbA} 1 \mathrm{c}$ and a reduction in the rate of nocturnal, confirmed hypoglycaemia was demonstrated in heterogeneous populations of patients diagnosed with diabetes. ${ }^{27-29}$

\section{Conclusion}

The longer-acting basal analogues insulins, IDeg and Gla-300, offer the prescriber a wider armamentarium of treatment for uncontrolled hyperglycaemia with PD profiles with smaller gradients than current basal insulins. However, the effect of the more evenly distributed insulin release on the incidence of hypoglycaemia of these insulins cannot be concluded from the glucose clamp studies at hand: pivotal clinical trial evidence remains the gold standard. Armed with a scientifically sound understanding of the pharmacokinetic and pharmacodynamic properties of new longer-acting basal analogue insulins, the treating doctor can utilise these properties to the advantage of the patient diagnosed with diabetes.

Conflict of interest - Dr K. Naidu has previously received honoraria from Sanofi. Dr Mothilal is the Medical Director, Dr McMaster is the Medical Adviser and Dr A. van Tonder is a Medical Science Liaison at Sanofi-Aventis South Africa (Pty) Ltd, a member of the SANOFI Group.

\section{References}

1. Amod A, Motala A, Levitt $N$, et al. The 2012 SEMDSA guideline for the management of type 2 diabetes. JEMDSA. 2012;17(1): S1-S94.

2. Eliaschewitz FG, Barreto T. Concepts and clinical use of ultra-long basal insulin. Diabetology and Metabolic Syndrome. 2016;8(2): 1-8.

3. Hilgenfeld R, Seipke $G$, Berchtold $H$, et al. The evolution of insulin glargine and its continuing contribution to diabetes care. Drugs. 2014;74: 911-27. https://doi. org/10.1007/s40265-014-0226-4

4. Bolli GB, Owens DR. Insulin glargine. The Lancet. 2000;356(9228): 443-5. https:// doi.org/10.1016/S0140-6736(00)02546-0

5. Steinstraesser A, Schmidt R, Bergmann $K$, et al. Investigational new insulin glargine $300 \mathrm{U} / \mathrm{ml}$ has the same metabolism as insulin glargine $100 \mathrm{U} / \mathrm{ml}$. Diabetes Obes Metab. 2014;16(9): 873-876. https://doi.org/10.1111/dom.12283

6. Heinemann L. Time-action profiles of insulin preparations. Mainz: Kirchheim; 2004.

7. Heise T, Nosek L. Bottcher SG, at al. Ultra-long-acting insulin degludec has a flat and stable glucose-lowering effect in type 2 diabetes. Diabetes Obes Metab. 2012;14: 944-950. https://doi.org/10.1111/dom.2012.14.issue-10

8. Heise T, Hermanski L, Nosek L, et al. Insulin degludec: four times lower pharmacodynamic variability than insulin glargine under steady-state conditionsintype 1 diabetes. Diabetes Obes Metab. 2012;14: 859-864. https:// doi.org/10.1111/dom.2012.14.issue-9

9. HeiseT,HövelmannU,NosekL,etal.Comparisonofthepharmacokinetic and pharmacodynamics profiles of insulin degludec and insulin glargine. Expert Opin Drug Metab Toxicol. 2015;11(8): 1193-1201. https://doi.org/10.1517/1742 5255.2015.1058779

10. Becker RHA, Dahmen R, Bergmann $K$, et al. New insulin glargine 300 units. $\mathrm{mL}-1$ provides a more even activity profile and prolonged glycaemic control at steady state compared with insulin glargine 100 units.mL-1. Diabetes Care. 2014;1-7.

11. ShiramotoM,EtoT,IrieS,etal.Single-dosenewinsulinglargine $300 \mathrm{U} / \mathrm{ml}$ provides prolonged,stableglycaemiccontrolinJapaneseandEuropean people with type 1 diabetes. Diabetes Obes Metab. 2015;17: 254-60. https://doi.org/10.1111/ dom.2015.17.issue-3

12. Becker RHA, Nowotny I, Teichert L, et al. Low within- and between- day variability in exposure to new insulin glargine $300 \mathrm{U} / \mathrm{ml}$. Diabetes Obes Metab. 2015;17: 261-7. https://doi.org/10.1111/dom.2015.17. issue-3

13. Swinnen SGHA, Holleman F, DeVries JH. The interpretation of glucose clamp studies of long-acting insulin analogues: from physiology to marketing and back. Diabetologia. 2008;51: 1790-5. https://doi. org/10.1007/ s00125-008-1098-5

14. Lepore $M$, Pampanelli $S$, Fanelli $C$, et al. Pharmacokinetics and pharmacodynamics of subcutaneous injection of long-acting human insulin analogue Glargine, NPH insulin and Ultralente human insulin and continuous subcutaneous infusion of insulin lispro. Diabetes. 2000; 49:2142 Home P. Pharmacokinetics and pharmacodynamics of biosimilar insulins: is clamp technology fit for purpose? Diabetes Care. 2015;38: 2234-36.

15. Home P. Pharmacokinetics and pharmacodynamics of biosimilar insulins: is clamp technology fit for purpose? Diabetes Care. 2015;38: 2234-2236. https:// doi.org/10.2337/dc15-1046 
16. Heise T, Zijlstra E, Nosek L, et al. Euglycaemic glucose clamp: what it can and cannot do, and how to do it. Diabetes Obes Metab 2016;18(10): 962-972. https:// doi.org/10.1111/dom.2016.18.issue-10

17. Siraj EJ, Rubin DJ, Riddle MC, et al. Insulin dose and cardiovascular mortality in the ACCORD trial. Diabetes Care. 2015;38: 2000-8. https://doi.org/10.2337/ dc15-0598

18. Rave K, Bott S, Heinemann L, et al. Time-Action Profile of Inhaled Insulin in Comparison With Subcutaneously Injected Insulin Lispro and Regular Human Insulin. Diabetes Care. 2005;28: 1077-82. https://doi.org/10.2337/ diacare.28.5.1077

19. Bailey T, Dahmen, R, Petthus J, et al. Insulin glargine $300 \mathrm{U} / \mathrm{ml}$ (Gla-300) provides more stable and more evenly distributed steady-state PK/PD profiles compared with insulin degludec in type 1 diabetes. In: Diabetes Techology Society; 2016 10-12 November 2016, Bethesda, MD, USA.

20. Kurtzhals $P$, Havelund $S$, Jonassen $I$, et al. Albumin binding of insulins acylated with fatty acids: characterization of the ligand- protein interaction and correlation between binding affinity and timing of the insulin effect in vivo. Biochem. J. 1995;312(3): 725-31. https://doi.org/10.1042/bj3120725

21. Jonassen I, Havelund $S$, Hoeg-Jensen $T$, et al. Design of the novel protraction mechanism of insulin degludec, an ultra- long-acting basal insulin. Pharm Res. 2012;29: 2104-24. https://doi.org/10.1007/s11095-012-0739-z

22. Lamos EM, Younk LM, Davis SN. Concentrated insulins: the new basal insulins. Ther Clin Risk Manag. 2016;12: 389-400.

23. Weyer C, Hanson RL, Tataranni PA., et al. A high fasting plasma insulin concentration predicts type 2 diabetes independent of insulin resistance: evidence for a pathogenic role of relative hyperinsulinemia. Diabetes. 2000;49: 2094-2101. https://doi.org/10.2337/diabetes.49.12.2094
24. Heller S, Buse J, Fisher M, et al. Insulin degludec, an ultra-long acting basa insulin, versus insulin glargine in basal-bolus treatment with mealtime insulin aspart in type 1 diabetes (BEGIN Basal-Bolus Type 1): a phase 3, randomised, open-label, treat-to-target non-inferiority trial. Lancet. 2012;379: 1489-97. https://doi.org/10.1016/S0140-6736(12)60204-9

25. Garber AJ, King $A B$, Prato $S$, et al. Insulin degludec, an ultra- long acting basal insulin versus insulin glargine in basal-bolus treatment with mealtime insulin aspart in type 2 diabetes (BEGIN Basal-Bolus Type 2): a phase 3, randomised, open-label, treat- to-target non-inferiority trial. Lancet. 2012;379: 1498-1507. https://doi.org/10.1016/S0140-6736(12)60205-0

26. Zinman B, Philis-Tsimikas A, Cariou B, et al. Insulin degludec versus insulin glargine in insulin-naïve patients with type 2 diabetes: a 1-year randomised, treat-to-target trial (BEGIN Once Long). Diabetes Care. 2012;35(12): 2464-71. https://doi.org/10.2337/dc12-1205

27. Riddle $M C$, Bolli GB, Ziemen $M$, et al. New insulin glargine 300 units $/ \mathrm{ml}$ versus glargine 100 units $/ \mathrm{ml}$ in people with type 2 diabetes using basal and mealtime insulin: glucose control and hypoglycaemia in a 6-month randomised controlled trial (EDITION 1). Diabetes Care. 2014;37: 2755-62. https://doi.org/10.2337/dc14-0991

28. Yki-Järvinen $H$, Bergenstal $R$, Ziemen $M$, et al. New insulin glargine 300 units/ $\mathrm{ml}$ versus glargine 100 units/ml in people with type 2 diabetes using oral agents and basal insulin: glucose control and hypoglycaemia in a 6-month randomised controlled trial (EDITION 2). Diabetes Care. 2014;37: 3235-43. https://doi.org/10.2337/dc14-0990

29. Home PD, Bergenstal RM, Bolli GB, et al. New insulin glargine 300 units/ml versus glargine 100 units $/ \mathrm{ml}$ in people with type 1 diabetes: a randomised, phase $3 a$, open-label clinical trial (EDITION 4). Diabetes Care. 2014;7(12): 3235-43. 